\title{
ON THE PRODUCT OF SELF-ADJOINT STURM-LIOUVILLE DIFFERENTIAL OPERATORS IN DIRECT SUM SPACES
}

\author{
SOBHY EL-SAYED IBRAHIM
}

\begin{abstract}
In this paper, the second-order symmetric Sturm-Liouville differential expressions $\tau_{1}, \tau_{2}, \ldots, \tau_{n}$, with real coefficients on any finite number of intervals are studied in the setting of the direct sum of the $L_{w}^{2}$-spaces of functions defined on each of the separate intervals. It is shown that the characterization of singular self-adjoint boundary conditions involves the sesquilinear form associated with the product of Sturm-Liouville differential expressions and elements of the maximal domain of the product operators, it is an exact parallel of that in the regular case. This characterization is an extension of those obtained in [6], [7], [8], [9], [12], [14] and [15].
\end{abstract}

\section{Introduction}

In [7] Everitt and Zettl studied the boundary value problem for Sturm-Liouville differential expressions

$$
\tau_{r}[y]=-\left(p_{r} y^{\prime}\right)^{\prime}+q_{r} y \text { on } I_{r}=\left(a_{r}, b_{r}\right), \quad-\infty \leq a_{r}<b_{r} \leq \infty ; r=1,2,
$$

with real-valued Lebesgue measurable functions $p_{r}, q_{r}, w_{r}$ from $I_{r}$ into $\mathbb{R}$ satisfying the following basic conditions :

$$
p_{r}^{-1}, q_{r}, w_{r} \in L_{l o c}\left(I_{r}\right), \quad w_{r}>0, \text { a.e., } r=1,2,
$$

on two intervals in the setting of the direct sum of the $L^{2}$-spaces of functions defined on each of the separate intervals, and in [9] S. E. Ibrahim extended this problem for any finite number of intervals. In the one interval case, the characterization of the singular self-adjoint boundary conditions for Sturm-Liouville problems is identical to that in the regular case provided that $y$ and $p y^{\prime}$ are replaced by certain Wronskians involving $y$ and two linearly independent solutions of $\tau[y]=0$ has been proved by Krall and Zettl in [12].

The relationship between the deficiency index of a symmetric differential expression (1.1) and its powers $\tau^{2}, \tau^{3}, \ldots$ has recently been studied by Chaudhuri and Everitt [1] and the relationship between the number of linearly independent $L^{2}(0, \infty)$ solutions of the equations $\tau_{j}[y]=0$ and of the product equations $\left(\tau_{1} \tau_{2} \cdots \tau_{n}\right) y=0$ has been investigated

Received August 9, 2004; revised May 25, 2005.

2000 Mathematics Subject Classification. Primary 34A05, 34B24, 47A55, 47E05.

Key words and phrases. Sturm-Liouville differential expressions, product differential expressions, deficiency indices, regular and singular end-points, integrable square solutions, direct sum spaces, boundary conditions. 
by Everitt [6]. These results are extension of those recently obtained in $[5,16,17,18]$ for the special case $\tau_{j}=\tau$ for $j=1, \ldots, n$, and $\tau$ is a real second-order symmetric differential expression.

Our objective in this paper is to show in the direct sum of the $L_{w}^{2}$-spaces of functions defined on each of the separate intervals that, the characterization of singular self-adjoint boundary conditions is identical to that in the regular case provided that $y$ and its quasiderivatives are replaced by sesquilinear forms associated with the product of SturmLiouville differential expressions, involving $y$ and elements of the maximal domain of the product operators. This characterization is an extentsion of those by Everitt and Zettl [6] and $[7,8,9,12,13,14,15]$ to the case of product Sturm-Liouville differential expressions $\tau_{1}, \tau_{2}, \ldots, \tau_{n}$ on any finite number of intervals $I_{r}=\left(a_{r}, b_{r}\right), r=1,2, \ldots, N$. Here the interior singularities occur only at the ends of the intervals. The operators involved are closed symmetric with Property (C) given below and direct sum of product operators $\prod_{j=1}^{n}\left[T_{0}\left(\tau_{j r}\right)\right], r=1,2, \ldots, N$,

$$
T_{0}\left(\tau_{1} \tau_{2} \cdots \tau_{n}\right)=\prod_{j=1}^{n} T_{0}\left(\tau_{j}\right)=\bigoplus_{r=1}^{N}\left(\prod_{j=1}^{n}\left[T_{0}\left(\tau_{j r}\right)\right]\right)
$$

In the regular case, these conditions can be interpreted as linear combinations of the values of the unknown function $y$ and its quasi-derivatives at the end-points $a_{r}$ and $b_{r}$, $r=1,2, \ldots, N$.

In the singular case, these conditions are given in terms of sesquilinear forms involving $y$ and linearly independent solutions of the product equation $\left(\tau_{1} \tau_{2} \cdots \tau_{n}\right) y=0$ which given by Everitt and Zettl in [6].

\section{Preliminaries}

We begin with a brief summary of adjoin pairs of operators and products operators, a full treatment may be found in [2, Chapter III], [5], [6], [7], [9], [10] and [11].

The domain and range of a linear operator $T$ acting in a Hilbert space $H$ will be denoted by $D(T)$ and $R(T)$ respectively and $N(T)$ will denote its null space. The nullity of $T$, written $\operatorname{nul}(T)$, is the dimension of $N(T)$ and the deficiency of $T$, written $\operatorname{def}(T)$, is the co-dimension of $R(T)$ in $H$; thus if $T$ is densely defined and $R(T)$ is closed, then $\operatorname{def}(T)=\operatorname{nul}\left(T^{*}\right)$. The Fredholm domain of $T$ is (in the notation of [2]) the open subset $\triangle_{3}(T)$ of $\mathbb{C}$ consisting of those values $\lambda \in \mathbb{C}$ which are such that $(T-\lambda I)$ is a Fredhom operator, where $I$ is the identity operator on $H$. Thus, $\lambda \in \triangle_{3}(T)$ if and only if $(T-\lambda I)$ has closed range and finite nullity and deficiency.

A closed operator $A$ in a Hilbert space $H$ has Property (C), if it has closed range and $\lambda=0$ is not an eigenvalue; i.e., there is some postive number $r$ such that $\|A x\| \geq r\|x\|$ for all $x \in D(A)$.

Note that, Property (C) is equivalent to $\lambda=0$ being a regular type point of $A$. This in turn is equivalent to the existence of $A^{-1}$ as a bounded operator on the range of $A$ (which need not be all of $H$ ). 
Given two operators $A$ and $B$, both acting in a Hilbert space $H$, we wish to consider the product operator $A B$. This is defined as follows:

$$
D(A B)=\{x \in D(B) \mid B x \in D(A)\} \text { and }(A B) x=A(B x) \text { for all } x \in D(A B) .
$$

It may happen in general that $D(A B)$ contains only the null element of $H$. However, in the case of many differential operators the domains of the product will be dense in $H$.

The next result gives conditions under which the deficiency of a product is the sum of the deficiencies of the factors.

Lemma 2.1.(cf. [6 Theorem A] and [17]) Let $A$ and $B$ be closed operators with dense domains in a Hilbert space $H$. Suppose that $\lambda=0$ is a regular type point for both operators and $\operatorname{def} A$ and $\operatorname{def} B$ are finite. Then $A B$ is a closed operator with dense domain, has $\lambda=0$ as a regular type point and

$$
\operatorname{def} A B=\operatorname{def} A+\operatorname{def} B .
$$

Evidently Lemma 2.1 extends to the product of any finite number of operators $A_{1}, A_{2}, \ldots, A_{n}$.

Let the interval $I_{r}, r=1,2, \ldots, N$ have end-points $a_{r}, b_{r}\left(-\infty \leq a_{r}<b_{r} \leq \infty\right)$, and let $w_{r}: I_{r} \rightarrow \mathbb{R}$ be a non-negative weight function with $w_{r} \in L_{l o c}^{1}\left(I_{r}\right)$ and $w_{r}(x)>0$ (for almost all $\left.x \in I_{r}\right)$. Then $H_{r}=L_{w_{r}}^{2}\left(I_{r}\right)$ denotes the Hilbert function space of equivalence classes of Lebesgue measurable functions such that $\int_{I} w_{r}|f|^{2}<\infty$; the inner-product is defined by:

$$
(f, g)_{r}:=\int_{I} w_{r}(x) f(x) \overline{g(x)} d x\left(f, g \in L_{w_{r}}^{2}\left(I_{r}\right)\right), \quad r=1,2, \ldots, N .
$$

We shall consider the Sturm-Liouville differential equation of the form:

$$
\tau_{r}[u]=-\left(p_{r} u^{\prime}\right)^{\prime}+q_{r} u=\lambda w_{r} u \text { on } I_{r}, \quad r=1,2, \ldots, N
$$

where the real-valued Lebesque measurable functions $p_{r}, q_{r}$ and $w_{r}$ from $I_{r}$ into $\mathbb{R}$ satisfying the conditions (1.2) which are taken to hold throughout this paper. Under this assumptions, $\tau_{r}$ is interpreted as a quasi-differential expressions, $u$ being a solution of (2.2) if $u$ and $p_{r} u^{\prime}$ are in $A C_{l o c}\left(a_{r}, b_{r}\right)$, the space of functions which are absolutely continuous on compact subsets of $\left(a_{r}, b_{r}\right)$, and (2.1) is satisfied almost everywhere on $\left(a_{r}, b_{r}\right)$. Also, $p_{r} u^{\prime}=u^{[1]}$ is called the quasi-derivative of $u$.

The equation (2.2) is said to be regular at the left end-point $a_{r} \in \mathbb{R}$, if for all $X \in\left(a_{r}, b_{r}\right)$,

$$
a_{r} \in \mathbb{R} ; p_{r}^{-1}, q_{r}, w_{r} \in L^{1}\left(a_{r}, X\right), \quad r=1,2, \ldots, N .
$$

Otherwise (2.2) is said to be singular at $a_{r}$. If (2.2) is regular at both end-points $a_{r}$ and $b_{r}$, then it is said to be regular; in this case we have,

$$
a_{r}, b_{r} \in \mathbb{R} ; p_{r}^{-1}, q_{r}, w_{r} \in L^{1}\left(a_{r}, b_{r}\right), \quad r=1,2, \ldots, N .
$$


We shall be concerned with the second-order symmetric differential expressions $\left(\tau_{r}=\right.$ $\tau_{r}^{+}$) on $I_{r}$ and when both end-points $a_{r}$ and $b_{r}$ may be either regular or singular endpoints of (2.2). Note that, in view of (1.2), an end-point of $I_{r}$ is regular for (2.2), if and only if it is regular for the equation,

$$
\tau_{r}^{+}[v]=\bar{\lambda} w_{r} v \quad(\lambda \in \mathbb{C}) \text { on } I_{r}, \quad r=1,2, \ldots, N
$$

where $\tau_{r}^{+}$is the formal, or Lagrangian adjoint of $\tau_{r}$ given by:

$$
\tau_{r}^{+}[v]=-\left(p_{r} v^{\prime}\right)^{\prime}+q_{r} v \text { on } I_{r}, r=1,2, \ldots, N
$$

The maximal domain $D\left(\tau_{r}\right)$ is defined by,

$$
D\left(\tau_{r}\right):=\left\{f: f, p_{r} f^{\prime} \in A C_{l o c}\left(I_{r}\right) \text { and } w_{r}^{-1} \tau_{r}[f] \in L_{w}^{2}\left(a_{r}, b_{r}\right), r=1, \ldots, N\right\},
$$

is a subspace of $L_{w_{r}}^{2}\left(a_{r}, b_{r}\right)$. The maximal operator $T\left(\tau_{r}\right)$ is defined by,

$$
T\left(\tau_{r}\right) u:=w_{r}^{-1} \tau_{r}[u]\left(u \in D\left(\tau_{r}\right)\right), \quad r=1,2, \ldots, N
$$

It is well known that $D\left(\tau_{r}\right)$ is dense in $L_{w}^{2}\left(a_{r}, b_{r}\right)$; see [2], [9], [11], [12] and [19].

In the regular problem the minimal operator $T_{0}\left(\tau_{r}\right)$ is the restriction of $w_{r}^{-1} \tau_{r}[u]$ to the subspace:

$$
D_{0}\left(\tau_{r}\right):=\left\{u: u \in D\left(\tau_{r}\right), u^{[s-1]}\left(a_{r}\right)=u^{[s-l]}\left(b_{r}\right)=0, s=1,2\right\}
$$

The subspace $D_{0}\left(\tau_{r}\right)$ is dense and closed in $L_{w_{r}}^{2}\left(a_{r}, b_{r}\right)$; see [2], [15] and [19].

In the singular problem we first introduce the operator $T_{0}^{\prime}\left(\tau_{r}\right) ; T_{0}^{\prime}\left(\tau_{r}\right)$ being the restriction of $w_{p_{r}}^{-1} \tau_{r}[$.$] to the subspace:$

$$
D_{0}^{\prime}\left(\tau_{r}\right):=\left\{u: u \in D\left(\tau_{r}\right), \operatorname{supp} u \subset\left(a_{r}, b_{r}\right), r=1,2, \ldots, N\right\} .
$$

This operator is densely-defined and closable in $L_{w}^{2}\left(a_{r}, b_{r}\right)$; and we defined the minimal operator $T_{0}\left(\tau_{r}\right)$, to be its closure (see [2], [15] and [19, Section 5]). We denote the domain of $T_{0}\left(\tau_{r}\right)$ by $D_{0}\left(\tau_{r}\right)$. It can be shown that:

$$
u \in D_{0}\left(\tau_{r}\right) \Rightarrow u^{[s-1]}\left(a_{r}\right)=0, \quad(s=1,2 ; r=1,2, \ldots, N),
$$

whenever we assume $a_{r}$ to be regular end-point and $b_{r}$ to be singular end-point.

For $f, g \in D\left(\tau_{r}\right)$ and $\alpha, \beta \in I_{r}$, Green's formula is given by:

$$
\int_{\alpha}^{\beta}\left\{\tau_{r}[f] \bar{g}-f \overline{\tau_{r}[g]}\right\} d x=[f, g]_{r}(\beta)-[f, g]_{r}(\alpha),
$$

where,

$$
[f, g]_{r}(.):=f \bar{g}^{[1]}-f^{[1]} \bar{g}, \quad f, g \in D\left(\tau_{r}\right), r=1,2, \ldots, N
$$


For $f, g \in D\left(\tau_{r}\right)$, the limits $\lim _{\alpha \rightarrow a^{+}}[f, g]_{r}(\alpha)$ and $\lim _{\beta \rightarrow b^{-}}[f, g]_{r}(\beta)$ exist and are finite. These are denoted by $[f, g]_{r}\left(a_{r}\right)$ and $[f, g]_{r}\left(b_{r}\right)$, respectively.

For $f, g \in A C_{l o c}\left(a_{r}, b_{r}\right)$, let

$$
W_{r}(f, g)=f p g^{\prime}-g p f^{\prime} .
$$

Choose two solutions $\theta$ and $\phi$ of $\tau_{r}[u]=0$ satisfying,

$$
W_{r}(\theta, \phi)(x)=1 \text { for all } x \in I_{r}, r=1,2, \ldots, N \text {. }
$$

Clearly such $\theta$ and $\phi$ exist, i.e., they can be determined by the initial conditions:

$$
\theta(c)=1,\left(p_{r} \theta^{\prime}\right)(c)=1, \phi(c)=0,\left(p_{r} \phi^{\prime}\right)(c)=1 \text { for all } c \text { in } I_{r} .
$$

Note that, the sesquilinear form $[f, g]_{r}$, in (2.8) can be written as:

$$
\begin{aligned}
{[f, g]_{r} } & =f p_{r} \bar{g}^{\prime}-\bar{g} p_{r} f^{\prime} \\
& =\left(\bar{g}, p_{r} \bar{g}^{\prime}\right)\left(\begin{array}{cc}
0 & -1 \\
1 & 0
\end{array}\right)\left(\begin{array}{c}
f \\
p_{r} f^{\prime}
\end{array}\right) .
\end{aligned}
$$

From (2.9) and (2.10), we get

$$
\left(\begin{array}{cc}
0 & -1 \\
1 & 0
\end{array}\right)=-\left(\begin{array}{cc}
0 & -1 \\
1 & 0
\end{array}\right)\left(\begin{array}{cc}
\theta & \phi \\
p_{r} \theta^{\prime} & p_{r} \phi^{\prime}
\end{array}\right)\left(\begin{array}{cc}
0 & -1 \\
1 & 0
\end{array}\right)\left(\begin{array}{cc}
\theta & p_{r} \theta^{\prime} \\
\phi & p_{r} \phi^{\prime}
\end{array}\right)\left(\begin{array}{cc}
0 & -1 \\
1 & 0
\end{array}\right),
$$

and hence the sesquilinear form in (2.8) can also be written as:

$$
\begin{aligned}
{[f, g]_{r} } & =\left(W_{r}(\bar{g}, \theta), W_{r}(\bar{g}, \phi)\right)\left(\begin{array}{cc}
0 & -1 \\
1 & 0
\end{array}\right)\left(\begin{array}{l}
W_{r}(f, \theta) \\
W_{r}(f, \phi)
\end{array}\right) \\
& =W_{r}(\bar{g}, \phi) W_{r}(f, \phi)-W_{r}(\bar{g}, \theta) W_{r}(f, \phi) \\
& =\operatorname{def}\left(\begin{array}{ll}
W_{r}(f, \theta) & W_{r}(f, \phi) \\
W_{r}(\bar{g}, \theta) & W_{r}(\bar{g}, \phi)
\end{array}\right), \quad r=1,2, \ldots, N ;
\end{aligned}
$$

see [9] and [12].

Lemma 2.2. If for some $\lambda_{0} \in \mathbb{C}$, there are two linearly independent solutions of $\tau_{r}[u]=\lambda_{0} w_{r} u$ in $L_{w}^{2}\left(a_{r}, b_{r}\right)$. Then all solutions of $\tau_{r}[u]=\lambda w_{r} u$ are in $L_{w}^{2}\left(a_{r}, b_{r}\right)$ for all $\lambda \in \mathbb{C}$; see [2, Chapter 3] for more details.

Theorem 2.3.(cf. [2, Theorem 3.10.1]) Let $f \in L_{l o c}^{1}\left(a_{r}, b_{r}\right)$ and suppose that the conditions (1.2) are satisfied. Then given any complex numbers $c_{\tau, 0}$ and $c_{\tau, 1}$ and any $x_{0} \in\left(a_{r}, b_{r}\right)$ there exist a unique solution of $\tau_{r}[\phi]=f$ in $\left(a_{r}, b_{r}\right)$ which satisfies $\phi_{r}\left(x_{0}\right)=$ $c_{\tau, 0}, \phi_{r}^{[1]}\left(x_{0}\right)=c_{\tau, 1}, r=1, \ldots, N$.

A simple consequence of Theorem 2.3 is that the solution of (2.1) form a 2dimemsional vector space over $\mathbb{C}$. If $\left(\alpha_{0}, \alpha_{1}\right)$ and $\left(\beta_{0}, \beta_{1}\right)$ are linearly independent 
vectors in $\mathbb{C}^{2}$ then the solutions $\phi_{r, 1}(., \lambda), \phi_{r, 2}(., \lambda)$ of the equation (2.2) which satisfy $\phi_{r, 1}\left(x_{0}, \lambda\right)=\alpha_{r, 0}, \phi_{r, 1}^{[1]}\left(x_{0}, \lambda\right)=\alpha_{r, 1}, \phi_{r, 2}\left(x_{0}, \lambda\right)=\beta_{r, 0}, \phi_{r, 2}^{[1]}\left(x_{0}, \lambda\right)=\beta_{r, 1}$ for some $x_{0} \in\left(a_{r}, b_{r}\right), r=1,2, \ldots, N$ form a basis for the space of soultions of the equation (2.2).

Note that, an important distinction between a regular end-point and a singular endpoint is the fact that at a regular end-point $x_{0}$, all inital value problems $\phi_{r}\left(x_{0}, \lambda\right)=c_{r, 0}$, $\phi_{r}^{[1]}\left(x_{0}, \lambda\right)=c_{r, 1}, c_{r, 0}, c_{r, 1} \in \mathbb{C}$ have a unique solutions. This is not true when $x_{0}$ is singular end-point (see [2], [10] and [13]).

Assume that $a_{r}$ and $b_{r}$ are singular end-points. For any $\alpha_{r}$ and $\beta_{r}$ in the open interval $\left(a_{r}, b_{r}\right)$ and any $\lambda \in \mathbb{C}$, the conditions (1.2) imply that any solution $\phi_{r}$ of the equation $(2.2)$ is in $L_{w_{r}}^{2}\left(\alpha_{r}, \beta_{r}\right)$; (see [10], [12] and [20]). However, it is possible that such a $\phi_{r}$ does not belong to $L_{w_{r}}^{2}\left(a_{r}, b_{r}\right)$. If $\phi_{r}$ is in $L_{w_{r}}^{2}\left(a_{r}, \beta_{r}\right)$ for some $\beta_{r} \in\left(a_{r}, b_{r}\right)$, then this is true for all $\beta_{r}$ in $\left(a_{r}, b_{r}\right)$. If all solutions of $(2.2)$ are in $L_{w_{r}}^{2}\left(a_{r}, b_{r}\right)$ for some $\beta_{r}$ in $\left(a_{r}, b_{r}\right)$, then we say that $\tau_{r}[$.$] is in the limit-circle case at a_{r}$, or simply that $a_{r}$ is $L C$. Otherwise, $\tau_{r}[$.$] is in the limit-point case at a_{r}$ or $a_{r}$ is $L P$. Similarly, $b_{r}$ is $L C$ means that all solutions of (2.2) are in $L_{w_{r}}^{2}\left(\alpha_{r}, b_{r}\right), a_{r}<\alpha_{r}<b_{r}, r=1,2, \ldots, N$. This classificantion is independent of $\lambda$ in (2.2); (see [9], [12], [13] and [15]). Otherwise $b_{\tau}$ is $L P$. The limit-point, limit-circle terminology are used for historical reasons.

The classification of the self-adjoint extensions of $T_{0}\left(\tau_{r}\right)$ depends, in an essential way, on the deficiency index of $T_{0}\left(\tau_{r}\right)$. We briefly recall the definition of this notion for abstract symmetric operators in a seperable Hilbert space.

A linear operator $A_{r}$ from a Hilbert space $H_{r}$ into $H_{r}$ is said to be symmetric if its domain $D\left(A_{r}\right)$ is dense in $H_{r}$ and $\left(A_{r} f, g\right)=\left(f, A_{r} g\right)$ for all $f, g \in D\left(A_{r}\right), r=1,2, \ldots, N$. Any such operator has associated with it a pair $\left(d_{r}^{+}, d_{r}^{-}\right)$, where each of $d_{r}^{+}, d_{r}^{-}$is a nonnegative or $+\infty$. The extended integers are called the deficiency indices of $A_{r}$ and we have the following:

For $\lambda \in \mathbb{C}$, the set of complex numbers, let $R_{\lambda}$ denote the range of $T_{0}\left(\tau_{r}\right)-\bar{\lambda} I$, $N_{\lambda, r}=R_{\lambda, r}^{\perp}$ and let

$$
N_{r}^{+}=N_{i, r}, N_{r}^{-}=N_{-i, r}, \quad i=\sqrt{-1}, r=1, \ldots, N
$$

$d_{r}^{+}=$dimension of $N_{r}^{+}$and $d_{r}^{-}=$dimension of $N_{r}^{-}$. The spaces $N_{r}^{+}, N_{r}^{-}$are called the deficiency spaces of $T_{0}\left(\tau_{r}\right)$ and $d_{r}^{+}, d_{r}^{-}$are called the deficiency indices of $T_{0}\left(\tau_{r}\right)$. These are related to the equation (2.2) as follows:

$$
N_{\lambda, r}=\left\{f \in D\left[T_{0}^{*}\left(\tau_{r}\right)\right] \mid\left[T_{0}^{*}\left(\tau_{r}\right)\right] f=\left[T\left(\tau_{r}\right)\right] f=w_{r}^{-1} \tau_{r}[f]=\lambda f, r=1, \ldots, N\right\} .
$$

Thus, $N_{r}^{+}, N_{r}^{-}$consist of the solutions of the equation (2.2) which lie in the space $H_{r}=L_{w_{r}}^{2}\left(I_{r}\right)$ for $\lambda=+i$ and $\lambda=-i$, respectively. Hence $d_{r}^{+}, d_{r}^{-}$are the number of linearly independent solutions of the equation (2.2) which are in the space $H_{r}$ for $\lambda=+i$ and $\lambda=-i$, respectively. It is clear for a symmetric differential operator $T_{0}\left(\tau_{r}\right)$ that:

$$
0 \leq d_{r}^{+}=d_{r}^{-} \leq 2, \quad r=1,2, \ldots, N
$$


We denote the common value by $d_{r}$ and call $d_{r}$ the deficiency index of $\tau_{r}$ on $I_{r}$. From the above discussion we see that there are only three possibilities for $d_{r}$ as: $d_{r}=0,1,2$, $r=1,2, \ldots, N$.

Note that, in the literature the maximal and minimal deficiency cases are often referred to as the limit-circle and limit-point cases. Strictly these latter terms are only suitable for the now classical second order differential expressions; in this case the terminology was originally introduced by Hermann Weyl. The term limit-point does give an acceptable description of the minimal deficiency case for real, and hence even-order, symmetric expressions.

Now, we recall the following results:

For any $\lambda \in \mathbb{C} \backslash \mathbb{R}$ and for a symmetric differential operator $T_{0}\left(\tau_{r}\right)$, we have from the general theory that,

$$
D\left(\tau_{r}\right)=D_{0}\left(\tau_{r}\right)+N_{r}^{+}+N_{r}^{-}, \quad r=1,2, \ldots, N
$$

where $D_{0}\left(\tau_{r}\right), N_{r}^{+}$and $N_{r}^{-}$are linearly independent subspaces and the sum is direct (which we indicate with the symbol + ); see [2], [7], [9], [11] and [15].

Any self-adjoint extension $S_{r}$ of the symmetric differential operator $T_{0}\left(\tau_{r}\right)$, satisfies

$$
T_{0}\left(\tau_{r}\right) \subset S_{r}=S_{r}^{*} \subset T_{0}^{*}\left(\tau_{r}\right), \quad r=1,2, \ldots, N,
$$

and hence is completely determined by specifying its domain $D\left(S_{r}\right)$,

$$
D_{0}\left(\tau_{r}\right) \subset D\left(S_{r}\right) \subset D\left(\tau_{r}\right), \quad r=1,2, \ldots, N .
$$

can be proved by using formula (2.13); (see [1], [2], [7], [9] and [10]).

Theorem 2.4. The operator $T_{0}\left(\tau_{r}\right)$ is a closed symmetric operator from $H_{r}$ into $H_{r}$ and

$$
T_{0}^{*}\left(\tau_{r}\right)=T\left(\tau_{r}\right) \text { and } T^{*}\left(\tau_{r}\right)=T_{0}\left(\tau_{r}\right), D_{0}\left(\tau_{r}\right)=\text { domain of } T^{*}\left(\tau_{r}\right), \quad r=1, \ldots, N \text {. }
$$

Proof. See [9], [12], [14] and [15, Section 17.4].

Some of the basic facts are summarized in:

Theorem 2.5.(cf. [12, Proposition 1])

(a) $D_{0}\left(\tau_{r}\right)=\left\{f \in D\left(\tau_{r}\right):[f, g]_{r}\left(b_{r}\right)-[f, g]_{r}\left(a_{r}\right)=0\right.$ for all $\left.f, g \in D\left(\tau_{r}\right)\right\}$.

(b) If $\tau_{r}[$.$] is in the limit point case at an end-point c$, then $[f, g]_{r}(c)=0$ for all $f, g \in$ $D\left(\tau_{r}\right), c=a_{r}$ or $c=b_{r}, r=1,2, \ldots, N$.

(c) If an end-point $c$ is regular, then for any solution $u, u$ and $u^{[1]}$ are continuous at $c$.

(d) If $a_{r}$ and $b_{r}$ are both regular end-points then for any $\alpha_{r}, \beta_{r}, \gamma_{r}$ and $\delta_{r}$ in $\mathbb{C}$, there exists a function $f$ in $D\left(\tau_{r}\right)$ such that

$$
\begin{aligned}
f\left(a_{r}\right)=\alpha_{r}, & f^{[1]}\left(a_{r}\right)=\beta_{r}, \\
f\left(b_{r}\right)=\gamma_{r}, & f^{[1]}\left(b_{r}\right)=\delta_{r}, \quad r=1,2 \ldots, N .
\end{aligned}
$$


(e) If $a_{r}$ is regular and $b_{r}$ is singular, then a function $f$ from $D\left(\tau_{r}\right)$ is in $D_{0}\left(\tau_{r}\right)$ if and only if the following conditions are satisfied:

(i) $f\left(a_{r}\right)=0, \quad f^{[1]}\left(a_{r}\right)=0$,

(ii) $[f, g]_{r}\left(b_{r}\right)=0$ for all $f, g \in D\left(\tau_{r}\right)$.

The analogous results hold when $a_{r}$ is singular and $b_{r}$ is regular; also see [8], [10] and $[12]$.

Lemma 2.6.(cf. [9] and [12, Lemma 2]) Given $\alpha_{r}, \beta_{r}, \gamma_{r}$ and $\delta_{r}$ in $\mathbb{C}$, then there exists a $\Psi \in\left[D\left(\tau_{r}\right) \backslash D_{0}\left(\tau_{r}\right)\right]$ such that

$$
\begin{aligned}
& W_{r}(\Psi, \theta)\left(a_{r}\right)=\alpha_{r}, \quad W_{r}(\Psi, \phi)\left(a_{r}\right)=\beta_{r}, \\
& W_{r}(\Psi, \theta)\left(b_{r}\right)=\gamma_{r}, \quad W_{r}(\Psi, \phi)\left(b_{r}\right)=\delta_{r}, \quad r=1,2, \ldots, N,
\end{aligned}
$$

where $\theta$ and $\phi$ are solutions of $\tau[u]=0$ satisfying (2.10). Furthermore, $\Psi$ can be taken to be a linear combination of $\theta$ and $\phi$ near each end-points.

Let $H$ be the direct sum

$$
H=\bigoplus_{r=1}^{N} H_{r}=\bigoplus_{r=1}^{N} L_{w_{r}}^{2}\left(a_{r}, b_{r}\right)
$$

The elements of $H$ will be denoted by $f=\left\{f_{1}, \ldots, f_{N}\right\}$ with $f_{1} \in H_{1}, \ldots, f_{N} \in H_{N}$.

Remark 2.7. When $I_{i} \cap I_{j}=\emptyset, i \neq j, i, j=1,2, \ldots, N$, the direct sum space $\bigoplus_{r=1}^{N}\left(I_{r}\right)$ can be natural identified with the space $L_{w_{r}}^{2}\left(\cup_{r=1}^{N} I_{r}\right)$, where $w=w_{r}$ on the interval $I_{r}, r=1, \ldots, N$. This remark is of particular significance when $\cup_{r=1}^{N} I_{r}$ may be taken as a single interval, see [8].

We now established by [7], [9] and [11] some further notation

$$
\begin{aligned}
D_{0}(\tau) & =\bigoplus_{r=1}^{N} D_{0}\left(\tau_{r}\right), \quad D_{0}(\tau)=\bigoplus_{r=1}^{N} D\left(\tau_{r}\right) \\
T_{0}(\tau) f & =\left\{T_{0}\left(\tau_{1}\right) f_{1}, \ldots, T_{0}\left(\tau_{N}\right) f_{N}\right\}, f_{1} \in D_{0}\left(T_{1}\right), \ldots, f_{N} \in D_{0}\left(\tau_{N}\right) .
\end{aligned}
$$

Also,

$$
T(\tau) f=\left\{T\left(\tau_{1}\right) f_{1}, \ldots, T\left(\tau_{N}\right) f_{N}\right\}, \quad f_{1} \in D\left(\tau_{1}\right), \ldots, f_{N} \in D\left(\tau_{N}\right) .
$$

The inner-product and sesquilinear form defined in (2.1) and (2.8) are:

$$
\begin{aligned}
(\underset{\sim}{f}, \underset{\sim}{g}) & =\sum_{r=1}^{N}\left(f_{r}, g_{r}\right), \\
{[\underset{\sim}{f}, \underset{\sim}{g}] } & =\sum_{r=1}^{N}\left\{\left[f_{r}, g_{r}\right]_{r}\left(b_{r}\right)-\left[f_{r}, g_{r}\right]_{r}\left(a_{r}\right), \quad \underset{\sim}{f}, \underset{\sim}{g} \in D(\tau),\right.
\end{aligned}
$$

where $\underset{\sim}{f}=\left\{f_{1}, \ldots, f_{N}\right\}, \underset{\sim}{g}=\left\{g_{1}, \ldots, g_{N}\right\}$. 
Note that $T_{0}\left(\tau_{r}\right)=\bigoplus_{r=1}^{N} T_{0}\left(\tau_{r}\right)$ is closed symmetric operator in $H$.

\section{The Product Operators in Direct Sum Spaces}

The proof of general theorem will be based on the results in this section. We start by listing some properties and results of Sturm-Liouville differential expressions $\tau_{1}, \tau_{2}, \ldots, \tau_{n}$, each of order two. For proofs the reader is referred to $[6],[9],[10],[16],[17]$ and $[18]$.

$$
\left\{\begin{array}{l}
\left(\tau_{1}+\tau_{2}\right)^{+}=\tau_{1}^{+}+\tau_{2}^{+} \\
\left(\tau_{1} \tau_{2}\right)^{+}=\tau_{2}^{+} \tau_{1}^{+},(\lambda \tau)^{+}=\bar{\lambda} \tau^{+} \text {for } \lambda \text { a complex number }
\end{array}\right\} .
$$

A consequence of Properties (3.1) is that if $\tau^{+}=\tau$ then $P(\tau)^{+}=P\left(\tau^{+}\right)$for $P$ any polynomial with complex coefficients. Also we note that the leading coefficients of a product is the product of the leading coefficients. Hence the product of regular differential expressions is regular. The next Lemma shows under conditions that the deficiency indices of a product is the sum of the deficiences of the factors.

Lemma 3.1.(cf. [6, Theorem 1]) Suppose $\tau_{j}$ is a regular differential expression on the interval $[a, b]$ such that the minimal operator $T_{0}\left(\tau_{j}\right)$ has Property $(\mathrm{C})$ for $j=1,2, \ldots, n$. Then

(i) The product operator $\prod_{j=1}^{n}\left[T_{0}\left(\tau_{j}\right)\right]$ is closed, have dense domain, Property $(\mathrm{C})$, and

$$
\operatorname{def}\left[\prod_{j=1}^{n} T_{0}\left(\tau_{j}\right)\right]=\sum_{j=1}^{n} \operatorname{def}\left[T_{0}\left(\tau_{j}\right)\right]
$$

also

(ii) $\left[T_{0}\left(\tau_{1} \tau_{2} \cdots \tau_{n}\right)\right] \subseteq \prod_{j=1}^{n}\left[T_{0}\left(\tau_{j}\right)\right]$

In part (ii) the containment may be proper, i.e., the operators $T_{0}\left(\tau_{1} \tau_{2} \cdots \tau_{n}\right)$ and $\prod_{j=1}^{n}\left[T_{0}\left(\tau_{j}\right)\right]$ are not equal in general.

Note that, for symmetric differential operator $T_{0}\left(\tau_{j}\right)$ which satisfies Property $(\mathrm{C})$ and by $(2.14)$, then $(3.2)$ is constant on $[0,2 n]$. In the problem with one singular end-point this constant is in $[n, 2 n]$, while in the regular problem it is equal $2 n$; see [2].

Lemma 3.2.(cf. [6, Theorem 2]) Let $\tau_{1}, \tau_{2}, \ldots, \tau_{n}$ be regular differential expressions on $[a, b]$. Suppose that $T_{0}\left(\tau_{j}\right)$ satisfies Property $(\mathrm{C})$ for $j=1,2, \ldots, n$. Then

$$
T_{0}\left(\tau_{1} \tau_{2} \cdots \tau_{n}\right)=\prod_{j=1}^{n} T_{0}\left(\tau_{j}\right)
$$

if and only if the following partial separation condition is satisfied: $\left\{f \in L_{w}^{2}(a, b), f^{[s-1]} \in\right.$ $A C_{\text {loc }}[a, b)$, where $s$ is the order of product expression $\left(\tau_{1} \tau_{2} \cdots \tau_{n}\right)$ and $\left(\tau_{1} \tau_{2} \cdots \tau_{n}\right)^{+} f \in$ $L_{w}^{2}(a, b)$ together imply that:

$$
\left.\left(\prod_{j=1}^{k}\left(\tau_{j}^{+}\right)\right) f \in L_{w}^{2}(a, b), \quad k=1, \ldots, n-1\right\} .
$$


Therefore (3.3) and (3.4) are equivalent.

We shall say that the product $\left(\tau_{1} \tau_{2} \cdots \tau_{n}\right)$ is a partially separated expressions in $L_{w}^{2}(a, b)$ whenever Property (3.4) holds.

Lemma 3.3. [cf. [6] and [10, Lemma 3.3] Let $\tau_{j}$ be a regular differential expression on $[a, b]$ for $j=1, \ldots, n$. If all solutions of the differential equation $\left(\tau_{j}\right) u=0$ and $\left(\tau_{j}^{+}\right) v=0$ on $[a, b]$ are in $L_{w}^{2}(a, b)$ for $j=1, \ldots, n$; then all solutions of $\left(\tau_{1} \tau_{2} \cdots \tau_{n}\right) u=0$ and $\left(\tau_{1} \tau_{2} \cdots \tau_{n}\right)^{+} v=0$ are in $L_{w}^{2}(a, b)$.

The special case of Lemma 3.3 when $\tau_{j}=\tau$ for $j=1,2, \ldots, n$ and $\tau$ is symmetric was established in [17]. In this case it is easy to see that the converse also holds. If all solutions of $\tau^{n} u=0$ are in $L_{w}^{2}(a, b)$ then all solutions of $\tau u=0$ must be in $L_{w}^{2}(a, b)$. In general, if all solutions of $\left(\tau_{1} \tau_{2} \cdots \tau_{n}\right) u=0$ are in $L_{w}^{2}(a, b)$ then all solutions of $\tau_{n} u=0$ are in $L_{w}^{2}(a, b)$ since these also solutions of $\left(\tau_{1} \tau_{2} \cdots \tau_{n}\right) u=0$. If all solutions of the adjoints equation $\left(\tau_{1} \tau_{2} \cdots \tau_{n}\right)^{+} v=0$ are also in $L_{w}^{2}(a, b)$ then it follows similarly that all solutions of $\tau_{1}^{+} v=0$ are in $L_{w}^{2}(a, b)$. So in particular for $n=2$ we have established the following Corollary.

Corollary 3.4. Suppose $\tau_{1}, \tau_{2}$ and $\tau_{1} \tau_{2}$ are all regular symmetric expressions on $[a, b)$. Then the product is in maximal deficiency case at $b$ if and only if both $\tau_{1}, \tau_{2}$ are in the maximal deficiency case at $b$ (i.e., if $\tau_{1}$ and $\tau_{2}$ are in the classical limit-circle case at $b$, then the fourth-order expression $\tau_{1} \tau_{2}$ is in the limit-circle case at $b\left(\right.$ i.e., $\left.d^{+}=d^{-}=4\right)$, we refer to $[6$, Corollary 2] for more details.

In connection with the application of Theorem 3.1 to get information about the deficiency indices of symmetric differential expressions, we note that the product of symmetric expressions is not symmetric in general. However, any power of a symmetric expression is symmetric and so called symmetric such as $\tau_{1} \tau_{2} \tau_{1}, \tau_{1} \tau_{2} \tau_{3} \tau_{2} \tau_{1}$, etc., of symmetric expressions are symmetric.

In the case of product operators in direct sum spaces, we summarize a few additional properties of $T_{0}(\tau)$ in the form of a lemma:

Lemma 3.5. Let $\tau_{1}, \tau_{2}, \ldots, \tau_{n}$ be regular differential expressions on $[a, b]$. Suppose that $T_{0}\left(\tau_{j}\right)$ satisfies Property $(\mathrm{C})$ for $j=1,2, \ldots, n$. Then:

(a) $\left[\prod_{j=1}^{n} T_{0}^{*}\left(\tau_{j}\right)\right]=\bigoplus_{r=1}^{N}\left[\prod_{j=1}^{n} T_{0}^{*}\left(\tau_{j r}\right)\right]=\bigoplus_{r=1}^{N}\left[\prod_{j=1}^{n} T\left(\tau_{j r}\right)\right]$.

In particular,

$$
D\left[\prod_{j=1}^{n} T_{0}^{*}\left(\tau_{j}\right)\right]=\bigoplus_{r=1}^{N} D\left[\prod_{j=1}^{n} T_{0}^{*}\left(\tau_{j r}\right)\right]=\bigoplus_{r=1}^{N} D\left[\prod_{j=1}^{n} T\left(\tau_{j r}\right)\right] .
$$

(b) $\operatorname{nul}\left[\prod_{j=1}^{n} T_{0}\left(\tau_{j}\right)\right]=\sum_{r=1}^{N} \operatorname{nul}\left[\prod_{j=1}^{n} T_{0}\left(\tau_{j r}\right)\right]=\sum_{r=1}^{N}\left(\sum_{j=1}^{n} \operatorname{nul}\left[T_{0}\left(\tau_{j r}\right)\right]\right)$.

(c) The deficiency index of $\prod_{j=1}^{n} T_{0}\left(\tau_{j}\right)$ is given by:

$$
\operatorname{def}\left[\prod_{j=1}^{n} T_{0}\left(\tau_{j}\right)\right]=\sum_{r=1}^{N} \operatorname{def}\left[\prod_{j=1}^{n} T_{0}\left(\tau_{j r}\right)\right]=\sum_{r=1}^{N}\left(\sum_{j=1}^{n} \operatorname{def}\left[T_{0}\left(\tau_{j r}\right)\right]\right) .
$$


The sesquilinear (bilinear) form $[f, g]$ can be written similar to that in (2.8) and (2.11) as follows: For $f, g \in D\left(\tau_{1} \tau_{2} \cdots \tau_{n}\right)$,

$$
\begin{aligned}
{[f, g](x) } & =\sum_{r=1}^{N}\left(\sum_{k=1}^{n}(-1)^{(k-1)}\left(f_{r}^{[k-1]} \bar{g}_{r}^{[2 n-k]}-f_{r}^{[2 n-k]}{\overline{g_{r}}}^{[k-1]}\right)_{r}(x)\right) \\
& =\sum_{r=1}^{N}\left(\left(\overline{g_{r}},{\overline{g_{r}}}^{[1]}, \ldots,{\overline{g_{r}}}^{[2 n-1]}\right)_{r} J_{2 n \times 2 n}\left(f_{r}, f_{r}^{[1]}, \ldots, f_{r}^{[2 n-1]}\right)_{r}^{\top}(x)\right) \\
& =\sum_{r=1}^{N}\left(\left[\overline{g_{r}}, \phi_{r, 1}\right], \ldots,\left[\overline{g_{r}}, \phi_{r, 2 n}\right]\right)_{r} J_{2 n \times 2 n}\left(\left[f_{r}, \phi_{r, 1}\right], \ldots,\left[f_{r}, \phi_{r, 2 n}\right]\right)_{r}^{\top}(x)
\end{aligned}
$$

$\top$ for transposed matrix, where $f_{r}^{[2 n-k]}, k=1, \ldots, 2 n ; r=1, \ldots, N$, are the quasi derivatives of $f_{r}, J_{2 n \times 2 n}=\left((-1)^{i} \delta_{r, 2 n+1-j}\right)(1 \leq i, j \leq 2 n)$ and $\phi_{r, 1}, \phi_{r, 2}, \ldots, \phi_{r, 2 n}$ are linearly independent solutions of the equation $\left[\prod_{j=1}^{n}\left(\tau_{j r}\right)\right] u=0, r=1, \ldots, N$. We refer to [9], [12] and [13] for more details.

Lemma 3.6. Let $\tau_{1}, \tau_{2}, \ldots, \tau_{n}$ be regular differential expressions on $[a, b]$. Suppose that $T_{0}\left(\tau_{j}\right)$ satisfies Property (C) for $j=1,2, \ldots, n$. Then

$$
T_{0}\left(\tau_{1} \tau_{2} \cdots \tau_{n}\right)=\prod_{j=1}^{n} T_{0}\left(\tau_{j}\right)=\bigoplus_{r=1}^{N}\left(\prod_{j=1}^{n}\left[T_{0}\left(\tau_{j r}\right)\right]\right) .
$$

Proof. The proof follows from (2.18), (2.20), (3.3), Lemma 3.2 and Lemma 3.5.

Note that, if $\prod_{j=1}^{n} S_{j r}, r=1, \ldots, N$ are self-adjoint extensions of $\prod_{j=1}^{n} T_{0}\left(\tau_{j r}\right)$, then by Lemma 3.6,

$$
S=\prod_{j=1}^{n} S_{j}=\bigoplus_{r=1}^{N}\left[\prod_{j=1}^{n} S_{j r}\right]
$$

is a self-adjoint extension of $T_{0}\left(\tau_{1} \tau_{2} \cdots \tau_{n}\right)$; see also [3] and [10].

The next result is a straightforward extension of Theorem 4 in [15, Section 18.1]; see also [2], [8] and [9].

Theorem 3.7. If the operator $S=\bigoplus_{r=1}^{N}\left(\prod_{j=1}^{n} S_{j r}\right)$ with $D(S)$ is a self-adjoint extension of the minimal operator $T_{0}\left(\tau_{1} \tau_{2} \cdots \tau_{n}\right)=\bigoplus_{r=1}^{N}\left(\prod_{j=1}^{N}\left[T_{0}\left(\tau_{j r}\right)\right]\right.$ with def $\left[T_{0}\left(\tau_{1} \tau_{2}\right.\right.$ $\left.\left.\cdots \tau_{n}\right)\right]=d \in[0,2 n N]$, then there exist $\Psi_{1}, \ldots, \Psi_{d}$ in $D(S) \subset D\left[T\left(\tau_{1} \tau_{2} \cdots \tau_{n}\right)\right]$ satisfying the following conditions:

(i) $\Psi_{1}, \ldots, \Psi_{d}$ are linearly independent modulo $D\left[T_{0}\left(\tau_{1} \tau_{2} \cdots \tau_{n}\right)\right]$.

(ii) $\left[\Psi_{j}, \Psi_{k}\right]_{a}^{b}=0, j, k=1, \ldots, d$.

(iii) $D(S)$ consists precisely of those $y$ in $D\left[T\left(\tau_{1} \tau_{2} \cdots \tau_{n}\right)\right]$ which satisfy,

$$
\left[y, \Psi_{j}\right]_{a}^{b}=0, \quad j=1, \ldots, d .
$$


Conversely, given $\Psi_{1}, \ldots, \Psi_{d}$ in $D\left[T\left(\tau_{1} \tau_{2} \cdots \tau_{n}\right)\right]$ which satisfy (i) and (ii), the set $D(S)$ defined by (iii) is a self-adjoint domain.

Proof. The proof is entirely similar to that in [10], [12] and [15, Theorem 18.1.4] and therefore omitted.

Remark 3.8. It is well known from Natmark [15] that no boundary condition is needed at a limit-point end-point in order to get a self-adjoint realization of $\prod_{j=1}^{n}\left(\tau_{j}\right) u=$ 0 . If both end-points are $L P$, then no boundary conditions are necessary and hence the minimal (maximal) operator associated with $\prod_{j=1}^{n}\left(\tau_{j}\right)$ in $L_{w}^{2}(a, b)$ is itself self-adjoint and has no proper self-adjoint extensions (restrictions). On the other hand, a boundary condition is needed for each limit-circle end-point.

The self-adjoint extensions are determined by boundary conditions imposed at the end-points of the interval $I$. The type of these boundary conditions depends on the nature of the problem in the interval $I$.

Theorem 3.9. Let $\prod_{j=1}^{n} \tau_{j}$ be regular symmetric differential expression on the interval $[a, b]$. Then the boundary conditions determine the domain of self-adjoint extension $S=\bigoplus_{r=1}^{N}\left(\prod_{j=1}^{n} S_{j r}\right)$ of $T_{0}\left(\tau_{1} \tau_{2} \cdots \tau_{n}\right)$ is the set of functions $y \in D\left[T\left(\tau_{1} \tau_{2} \cdots \tau_{n}\right)\right]$ which are such that

$$
\sum_{r=1}^{N} M^{r} Y\left(a_{r}\right)+\sum_{r=1}^{N} N^{r} Y\left(b_{r}\right)=0
$$

where,

$$
M^{r}=\left(\alpha_{j k}^{r}\right)_{1 \leq j, k \leq 2 n}, \quad N^{r}=\left(\beta_{j k}^{r}\right)_{1 \leq j, k \leq 2 n} ; \quad r=1,2, \ldots, N,
$$

are $2 n \times 2 n$ matrices over $\mathbb{C}, Y()=.\left(y, y^{[1]}, \ldots, y^{[2 n-1]}\right)^{\top}(),. \top$ for transposed matrix, and $\alpha_{j k}^{r}, \beta_{j k}^{r}$ are complex numbers satisfying,

$$
M^{r} J\left(M^{r}\right)^{*}=N^{r} J\left(N^{r}\right)^{*}, \quad J_{2 n \times 2 n}=(-1)^{i} \delta_{i, 2 n+1-j}(1 \leq i, j \leq 2 n) .
$$

Conversely, if $S$ is self-adjoint extension of $T_{0}\left(\tau_{1} \tau_{2} \cdots \tau_{n}\right)$, then there exist $2 n \times 2 n$ matrices $M^{r}$ and $N^{r}$ over $\mathbb{C}$ such that the conditions (3.10) and (3.12) are satisfied and $D(S)$ is the set of functions $y \in D\left[T\left(\tau_{1} \tau_{2} \cdots \tau_{n}\right)\right]$ satisfying $(3.10)$.

Proof. Let the boundary conditions (3.10) and (3.12) be given. By Theorem 2.5, there are functions $\Psi_{1, r}, \ldots, \Psi_{2 n, r}$ in $D\left[T\left(\tau_{1} \tau_{2} \cdots \tau_{n}\right)\right]$ which satisfy the conditions

$$
\begin{aligned}
& \bar{\Psi}_{j r}^{[2 n-k]}\left(a_{r}\right)=(-1)^{k} \alpha_{j k}^{r}, \\
& \bar{\Psi}_{j r}^{[2 n-k]}\left(b_{r}\right)=(-1)^{(k-1)} \beta_{j k}^{r}, \quad j, k=1, \ldots, 2 n, r=1,2, \ldots, N .
\end{aligned}
$$

Given (3.13), it is not difficult to show that (3.12) and (3.10) can be restated in the forms (3.8) and (3.9) respectively. It then follows from Theorem 3.7 that the domain determined by (3.10) and (3.12) is the domain of self-adjoint extension of $T_{0}\left(\tau_{1} \tau_{2} \cdots \tau_{n}\right)$.

Conversely, if $S$ is self-adjoint extension of $T_{0}\left(\tau_{1} \tau_{2} \cdots \tau_{n}\right)$, then by Theorem 3.7, $D(S)$ is determined by the functions $\Psi_{1, r}, \ldots, \Psi_{2 n, r}$ in $D\left[T\left(\tau_{1} \tau_{2} \cdots \tau_{n}\right)\right]$ satisfying (3.8) 
and (3.9). If $\alpha_{j k}^{r}$ and $\beta_{j k}^{r}, 1 \leq j, k \leq 2 n$ are then defined by (3.13), it is clear that $D(S)$ is determined by (3.10) and (3.12); see [9], [10] and [12] for more details.

In the following cases, the self-adjoint extension $S$ of $T_{0}\left(\tau_{1} \tau_{2} \cdots \tau_{n}\right)$ is determined by boundary conditions in terms of certain Wronskians (sesquilinear forms) involving $y$ and $2 n N$ linearly independent solutions of the equation $\left(\prod_{j=1}^{n} \tau_{j}\right) u=0$ at the singular end-points.

Case (i). Assume both end-points $a_{r}$ and $b_{r}$ are singular $L C$. By (3.5), (3.8) and Lemma 2.6, if we put,

$$
\begin{aligned}
{\left[\bar{\Psi}_{j}, \phi_{k, r}\right]\left(a_{r}\right) } & =(-1)^{k} \alpha_{j k}^{r}, \\
{\left[\bar{\Psi}_{j r}, \phi_{k, r}\right]\left(b_{r}\right) } & =(-1)^{(k-1)} \beta_{j k}^{r}, \quad j, k=1, \ldots, 2 n ; r=1,2, \ldots, N,
\end{aligned}
$$

then the boundary conditions of the function $y \in D\left[T\left(\tau_{1} \tau_{2} \cdots \tau_{n}\right)\right]$ have the same form (3.10), where $M^{r}, N^{r}$ satisfy (3.11) and (3.12), and $Y()=.\left(\left[y, \phi_{1, r}\right], \ldots,\left[y, \phi_{2 n, r}\right]\right)^{\top}($.$) .$

Case (ii). (a) Assume the left end-point $a_{r}$ is regular and the right end-point $b_{r}$ is singular $L C$. Then the boundary conditions of the functions $y \in D\left[T\left(\tau_{1} \tau_{2} \cdots \tau_{n}\right)\right]$ in this case are given by $(3.10)$, where

$$
\begin{aligned}
& Y\left(a_{r}\right)=\left(y, y^{[1]}, \ldots, y^{[2 n-1]}\right)^{\top}\left(a_{r}\right) \text { and } \\
& Y\left(b_{r}\right)=\left(\left[y, \phi_{1, r}\right], \ldots,\left[y, \phi_{2 n, r}\right]\right)^{\top}\left(b_{r}\right), \quad r=1,2, \ldots, N,
\end{aligned}
$$

and the matrices $M^{r}$ and $N^{r}$ satisfy (3.11).

(b) If the left end-point $a_{r}$ is singular $L C$ and the right end-point $b_{r}$ is regular, then let,

$$
Y\left(a_{r}\right)=\left(\left[y, \phi_{1, r}\right], \ldots,\left[y, \phi_{2 n, r}\right]\right)^{\top}\left(a_{r}\right) \text { and } Y\left(b_{r}\right)=\left(y, y^{[1]}, \ldots, y^{[2 n-1]}\right)^{\top}\left(b_{r}\right),
$$

and the rest is the same as in (a).

Case (iii). Assume one end-point is $L P$ end-point and the other is either regular or singular $L C$ end-point, then we have,

(a) Suppose $a_{r}$ is $L P$. Then the boundary conditions in this case on the functions $y \in D\left[T\left(\tau_{1} \tau_{2} \cdots \tau_{n}\right)\right]$ are $(3.10)$ with $M^{r}=0$; i.e.,

$$
\sum_{r=1}^{N} N^{r} Y\left(b_{r}\right)=0,
$$

where,

$Y\left(b_{r}\right)=\left(y, y^{[1]}, \ldots, y^{[2 n-1]}\right)^{\top}\left(b_{r}\right), \quad$ if $b_{r}$ is regular,

$Y\left(b_{r}\right)=\left(\left[y, \phi_{1, r}\right], \ldots,\left[y, \phi_{2 n, r}\right]\right)^{\top}\left(b_{r}\right) \quad$ if $b_{r}$ is singular and $L C, r=1,2, \ldots, N$.

(b) if $b_{r}$ is $L P$, then it suffices to reverse the roles of $a_{r}$ and $b_{r}$ in (a). 
Case (iv). If both end-points $a_{r}$ and $b_{r}, r=1,2, \ldots, N$ are $L P$, then no boundary conditions are necessary; see Remark 3.8 above.

\section{Discussion}

In this section, we show how Cases (i), (ii), (iii) and (iv) follow from the sesquilinear form (3.5), Lemma 2.6 and Theorem 3.7. The Cases $d=0, n N, 2 n N$ are considered separately.

Example 1. $d=0$. In this case, both end-points are $L P$ end-points and the minimal operator $T_{0}\left(\tau_{1} \tau_{2} \cdots \tau_{n}\right)$ is itself self-adjoint and has no proper self-adjoint extensions.

Example 2. $d=n N$. In this case, one end-point must be $L P$ and the other either regular or $L C$ end-point.

(2a) Assume $a$ is $L P$ and $b$ is regular. In this case Condition (iii) becomes,

$$
\begin{aligned}
{\left[y, \Psi_{j}\right]_{a}^{b} } & =\left[y, \Psi_{j}\right](b) \\
& =\sum_{r=1}^{N}\left(\sum_{k=1}^{n}(-1)^{(k-1)}\left[y^{[k-1]} \bar{\Psi}_{j r}^{[2 n-k]}-y^{[2 n-k]} \bar{\Psi}_{j r}^{[k-1]}\right]\right)\left(b_{r}\right)=0, \quad j=1, \ldots, n .
\end{aligned}
$$

If $b$ is regular, then $\Psi_{j r}\left(b_{r}\right), \Psi_{j r}^{[1]}\left(b_{r}\right), \ldots, \Psi_{j r}^{[2 n-1]}\left(b_{r}\right)$ can take an arbitrary values and so (3.10) can be rewritten as:

$$
\sum_{r=1}^{N} N^{r} Y\left(b_{r}\right)=0
$$

where $N^{r}=\left(\beta_{j k}^{r}\right)_{1 \leq j \leq n, 1 \leq k \leq 2 n}$ and $Y\left(b_{r}\right)=\left(y, y^{[1]}, \ldots, y^{[2 n-1]}\right)^{\top}\left(b_{r}\right), r=1, \ldots, N$.

From Theorem 3.7 (i), we have that not all of $\beta_{j, 1}, \ldots, \beta_{j, 2 n N}$ can be zero since this would imply by Theorem 3.7 that $\Psi_{j} \in D_{0}\left(\tau_{1} \tau_{2} \cdots \tau_{n}\right), j=1, \ldots, n N$. Condition (ii) becomes,

$$
\begin{aligned}
& N^{r} J_{2 n \times 2 n}\left(N^{r}\right)^{*}=0, \\
& J_{2 n \times 2 n}=(-1)^{i} \delta_{i, 2 n+1-j} \quad(1 \leq i, j \leq 2 n ; r=1, \ldots, N) .
\end{aligned}
$$

Hence, the self-adjoint "boundary conditions" are of the form (4.2) with real $\beta_{j, 1}^{r}, \ldots$, $\beta_{j, 2 n}^{r}$, not all zero $j=1, \ldots, n$.

We have similar result if $a$ is regular and $b$ is $L P$.

(2b) Assume $a$ is $L P$ and $b$ is $L C$. In this case, Condition (iii) becomes (4.1), which is equivalent to

$$
\sum_{r=1}^{N}\left(\left[\overline{\Psi_{j r}}, \phi_{1, r}\right], \ldots,\left[\overline{\Psi_{j r}}, \phi_{2 n, r}\right] J_{2 n \times 2 n}\left(\left[y, \phi_{1, r}\right], \ldots,\left[y, \phi_{2 n, r}\right]\right)^{\top}=0\right.
$$


$j=1, \ldots, n$. Set

$$
\left[\overline{\Psi_{j}}, \phi_{k, r}\right]\left(b_{r}\right)=(-1)^{(k-1)} \beta_{j k}^{r}, \quad j=1, \ldots, n ; k=1, \ldots, 2 n, r=1, \ldots, N .
$$

Then, the "boundary conditions" (iii) can be expressed as:

$$
\sum_{r=1}^{N} N^{r} Y\left(b_{r}\right)=0
$$

where $N^{r}=\left(\beta_{j k}^{r}\right)_{1 \leq j \leq n, 1 \leq k \leq 2 n}$ and $Y\left(b_{r}\right)=\left(\left[y, \phi_{1, r}\right], \ldots,\left[y, \phi_{2 n, r}\right]\right)^{\top}\left(b_{r}\right)$. Again by Theorem 3.7 (i), $\beta_{j, 1}^{r}, \ldots, \beta_{j, 2 n}^{r} j=1, \ldots, n$ are real and not all zero. Similarly for the case when $a$ is $L C$ and $b$ is $L P$.

Remark 4.1. Assume that $a$ is $L P$. Comparing (4.6) with (4.2), note that when $y^{[k-1]}\left(b_{r}\right)$ is replaced by $\left[y, \phi_{k, r}\right]\left(b_{r}\right), k=1, \ldots, 2 n, r=1, \ldots, N$, then the singular case when $b$ is $L C$ is an exact parallel to the case when $b$ is regular.

Example 3. $d=2 n N$. In this case, each end-point is either regular or $L C$. By (3.10), (3.13) and proceeding as in Case (2) above, we find that the condition (iii) is equivalent to the equations:

$$
\sum_{r=1}^{N}\left(\sum_{k=1}^{2 n} \alpha_{j k}^{r}\left[y, \phi_{k, r}\right]\left(a_{r}\right)+\sum_{k=1}^{2 n} \beta_{j k}^{r}\left[y, \phi_{k, r}\right]\left(b_{r}\right)\right)=0, \quad j=1, \ldots, 2 n .
$$

Theorem 3.7 (i) guarantee the linear independence of $2 n N$ equations in (4.7), and Condition (ii) reduces to the following conditions:

$$
\begin{aligned}
& \sum_{r=1}^{N}\left(\sum_{s=1}^{n} \alpha_{j k}^{r} \bar{\alpha}_{k, 2 n-s+1}^{r}-\sum_{s=1}^{n} \alpha_{j, 2 n-s+1}^{r} \bar{\alpha}_{k s}^{r}\right) \\
= & \sum_{r=1}^{N}\left(\sum_{s=1}^{n} \beta_{j s}^{r} \bar{\beta}_{k, 2 n-s+1}^{r}-\sum_{s=1}^{n} \beta_{j, 2 n-s+1}^{r} \bar{\beta}_{k s}^{r}\right), \quad j, k=1, \ldots, 2 n .
\end{aligned}
$$

We refer to [7], [8], [9] and [12] for more details.

Remark 4.2. It remains an open question as to characterize the singular non selfadjoint boundary conditions provided that $u$ and its quasi-derivatives are replaced by certain Wronskains (sesquilinear form) associated with non-symmetric differential expressions involving $u$ and elements of the maximal domain. 


\section{References}

[1] J. Chaudhuri and W. N. Everitt, On the square of a formaly self-adjoint differential expression, J. London Math. Soc. 1(1969), 661-673.

[2] D. E. Edmunds and W. D. Evans, Spectral Theory and Differential Operators, Oxford University Press, 1987.

[3] W. N. Everitt, A note on the self-adjoint domains of second-order differential equations, Quart. J. Math. (Oxford) 14(1963), 41-45.

[4] W. N. Everitt, Integrable square solutions of ordinary differential equations, Quart. J. Math. (Oxford) 14(1963), 170-180.

[5] W. N. Everitt and Giertz, On some properties of the powers of a formally self-adjoint differential expressions, Proc. London Math. Soc. 24(1972), 149-170.

[6] W. N. Everitt and Zettl, The number of integrable-square solutions of products of differential expression, Proc. Roy. Soc. of Edinburgh 76A(1977), 215-226.

[7] W. N. Everitt and A. Zettl, Sturm-Liouville differential operators in direct sum spaces, Rocky Mountain Journal of Mathematics 16(1986), 497-516.

[8] D. Hinton, A. M. Krall and K. Shaw, Boundary conditions for differential operators with intermediate deficiency index, Appl. Anal. 25(1987), 43-53.

[9] S. E. Ibrahim, On boundary conditions for Sturm-Liouville differential operators in the direct sum spaces, Rocky Mountain Journal of Mathematics 29(1999), 609-644.

[10] S. E. Ibrahim, On the domain of self-adjoint extension of the product of Sturm-Liouville differential operators, IJMMS 2003(2003), 695-709.

[11] Sobhy E. Ibrahim, The spectra of general differential operators in the direct sum spaces, Czechoslovak Mathematical Journal 54(2004), 9-29.

[12] A. M. Krall and A. Zettl, Singular self-adjoint Sturm-Liouville Problems, J. of Differential and Integral Equations I(1988), 423-432.

[13] A. M. Krall and A. Zettl, Singular self-adjoint Sturm-Liouville Problems II: Interior singular points, Saim J. Math. 19(1988), 1135-1141.

[14] S. J. Lee, On boundary conditions for ordinary linear differential operators, J. London Math. Soc. 12(1978), 447-454.

[15] M. A. Naimark, Linear Differential Operators, Part II, London, Harrap, 1968.

[16] A. Zettl, Deficiency indices of polynomials in symmetric differential expressions, Lecture Note in Mathematics 415 (Berlin:Springer, 1974), 293-301.

[17] A. Zettl, Deficiency indices of polynomials in symmetric differential expressions II, Proc. Roy. Soc. of Edinburgh Sect. A73(1975), 300-306.

[18] A. Zettl, The limit point and limit circle cases for polynomials in a differential operator, Proc. Roy. Soc. of Edinburgh Sect. A72(1975), 219-224.

[19] A. Zettl, Formally self-adjoint quasi-differential operators, Rocky Mountain Journal of Mathematics 5(1975), 453-474.

[20] P. W. Walker, A note on differential equations with all solutions of integrable-square, Pacific J. Math. 56(1975), 285-289.

Department of Mathematics, Faculty of Science, Benha University, P.O.Box 13518 Benha, Egypt.

E-mail: abomohamed@hotmail.com 